\title{
Surgical Treatment of the Static Perineal Modifications in Spinal Cord or Cauda Equina Lesions
}

\author{
F. Jurascheck, M.D., ${ }^{1}$ P. Dollfus, M.D., ${ }^{2}$ A. Chapuis, M.D., ${ }^{2}$ C. Schoe- \\ nahl, M.D., ${ }^{1}$ R. Fernandez, M.D., ${ }^{1}$ R. Al Salti, M.D. ${ }^{1}$ \\ ${ }^{1}$ Department of Urology, Hôpital Civil, 68057 Mulhouse Cedex, France, ${ }^{2}$ Centre \\ de Réadaptation, 57 rue Albert Camus, 68093 Mulhouse Cedex, France
}

\section{Summary}

Severe dysuria, due to insufficiency of the perineal floor associated during micturition with a posterior tilting of the prostate-bladder block in lower or in associated upper and lower motor neuron lesions, can be treated surgically by a prostato cytso pexy.

Since 1971, eight patients with post-trumatic conus and/or cauda equina lesions have been treated by this intervention. On the follow-up the satisfactory results appear to remain stable. An alternative surgical technique using the abdominal pyramidalis muscle is described so as to fix the prostate, associated with a bladderpexy. The recurrence of dysuria, after the intervention, has always been caused by an additional lower urinary tract pathology. The comfort of the patients has been greatly improved.

Key words: Spinal cord injury; Neuropathic bladder; Inactive perineum; Prostato-cysto-pexy.

\section{Introduction}

A functionally insufficient or inactive perineum, seen mostly with lower motor neuron lesions but also in mixed upper and lower motor neuron lesions, leads to a displacement of the pelvic perineal organs and, in time, to an increasing dysuria with urine retention and infection. Attempts to void by abdominal straining alone or associated with the Crede manoeuvre, patients with a neuropathic hypotonic of flaccid perineum, can provoke a lowering of the bladder and a backward tilting of the prostate. The only fixed element of the latter is the anchoring of its lower part to the pubis by the pubo uretralis ligament and the pars media of the fascia pelvis. In these conditions, the lower prostate bladder block is pushed backwards and downwards, forming two obstructive angles on the lower urinary tract: an anterior valve at the level of the internal sphincter and an obvious distorted kink between the prostate and the membranous urethra at the level of the uro-genital diaphragm. With high intra-abdominal pressure 

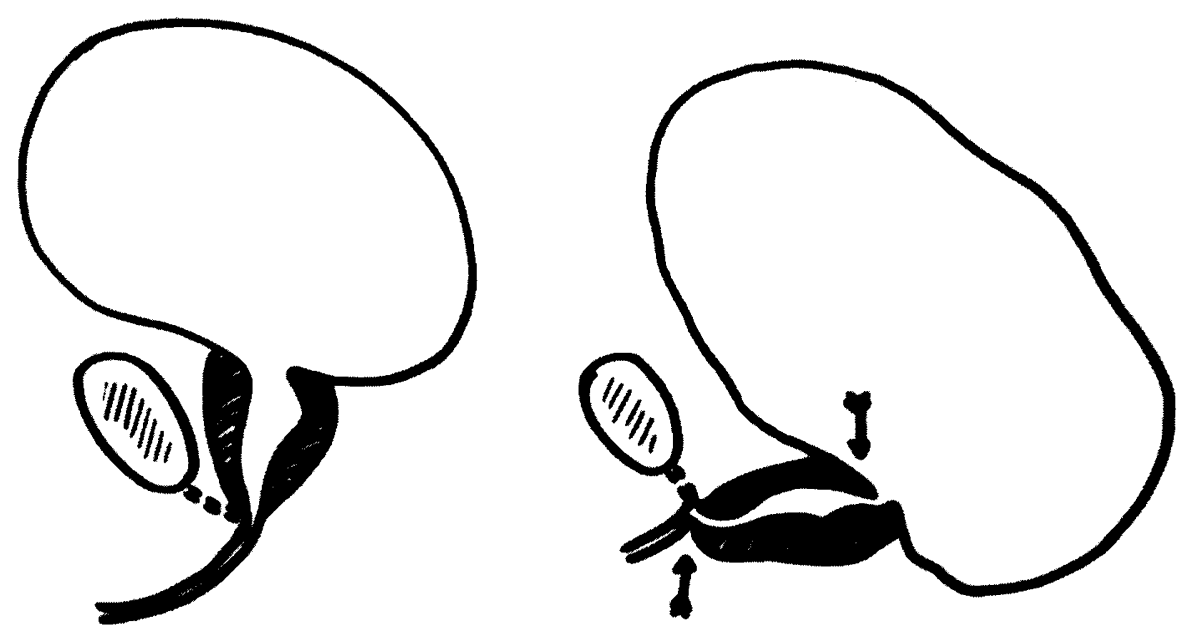

Figure 1 Shows the mechanism of the backward tilting of the prostate-bladder block occurring during strain demonstrating the obstructive mechanism.

this results in a narrow angle, open backwards and downwards. The mechanism of the phenomenon is very particular and rare in man. It has been described elsewhere (Dollfus et al., 1972) (Fig. 1). This functional obstruction is rather difficult to see on a normal micturition x-ray but can be easily detected using an oblique angle, under video, during its different phases, and can be recorded. The obstruction is best seen at the peak of abdominal strain.

In our first patient, we only understood what was happening by using a cineradiologic sequence during attempted micturition. It incited the urologist (F.J.) to help the patient by fixing the prostate to the pubis and the bladder to the abdominal wall (Fig. 2). Another patient was operated on elsewhere using the same technique but in the following cases we have modified the operative method by fixing the prostate to the musculus pyramidalis, the detached muscle being drawn backwards and downwards (Fig. 3) (Jurascheck et al., 1982). The functional results using the second method, appear to have improved the micturition pattern and the patient's comfort.

\section{Case reports}

We report four patients who have been followed clinically and radiologically for some time. Two other patients have been operated recently and two others, who have not been seen regularly, to our knowledge, have no recurrence of symptoms.

Case 1 Sq... S. (reported in our first publication in 1972): fracture of the L1 vertebra at the age of 45 , in 1970, causing complete paraplegia of the lower 


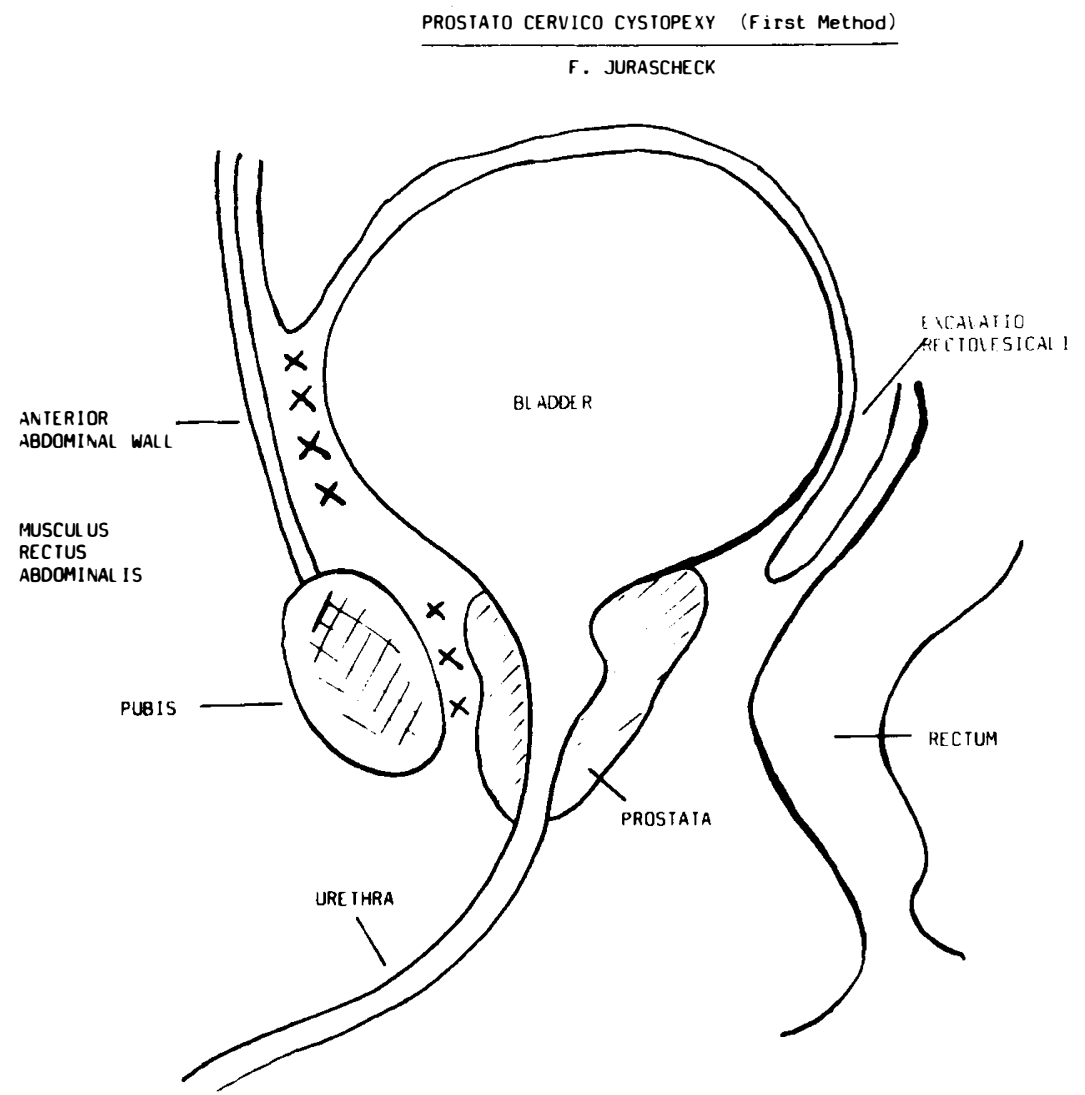

Figure 2 First method showing the pexy of the prostate and the bladder.

motor neuron type below T10 on the right and T12 on the left. Intermittent catheterisation for four months. Bladder training by the Crede manoeuvre and abdominal strain was unsuccessful (residual urine: $250-300 \mathrm{ml}$ ). Resection of the bladder neck was performed six months after the accident, but because of the persistence of a high residual urine, a second resection of the internal sphincter did not result in any benefit and dysuria increased. Paradoxically, there was no obstruction when passing a $27 \mathrm{~F}$.Ch. catheter. With a cineradiographic study during attempted micturition, we noticed the tilting of the bladder prostate block backwards into the perineum, causing the double obstruction. An operation, a prostato-cysto-pexy, consisted of fixation with a non absorbable suture, of the prostate to the supra-pubic ligament, and the anterior part of the bladder to the posterior fascia of the anterior wall of the abdomen (1971). The result was a dramatic improvement in micturition, low residuals, with a flow of 7 to $8 \mathrm{ml}$ per second. Few urinary infections occurred and the bladder emptying was satisfactory. Three years later, a small prostatic adenoma was resected as it was causing a gradual obstruction; a good result was obtained. Five years later urethral dilation was performed for stenosis. The result of this conservative procedure was good, with minimal residual urine. Dynamic radiological exam- 

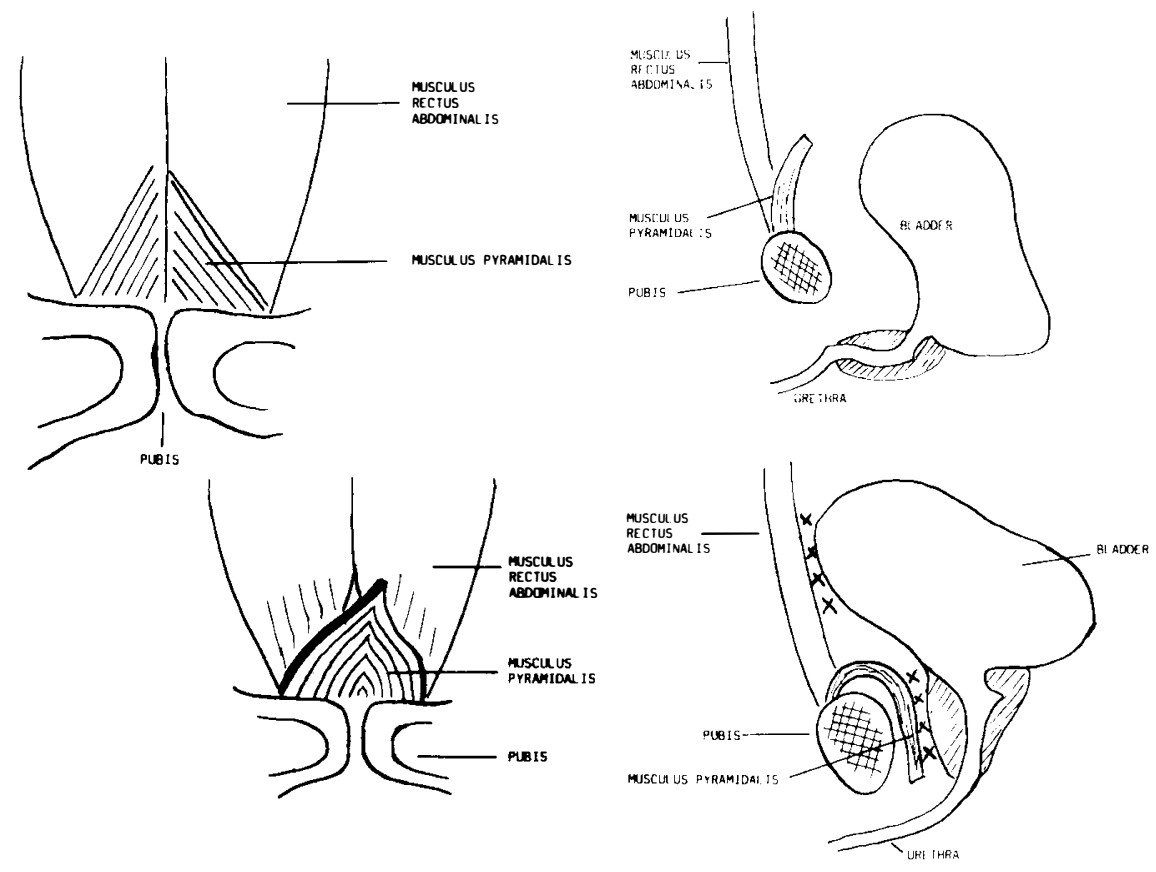

Figure 3 A, B The dissection of the

Figure 3 C, D Fixation of the prostate to pyramidalis muscle. the pyramidalis muscle and the bladder pexy.

inations showed that the fixation was still satisfactory (Fig. 4). This patient died from a cerebral haemorrhage eight years after the operation.

Case 2 De... G.: a 30 year old paraplegic patient with a fracture of T11, sustained in 1970, resulting in a complete flaccid paraplegia below T10 on the right, and $\mathrm{T} 9$ on the left. Treated by intermittent catheterisation, he was successfully bladder trained and micturition was obtained by abdominal straining within six months of the injury. No urological complications occurred during the following seven years but, progressively, after the 9th year, severe dysuria occurred causing incomplete slow micturition, lasting 20 minutes, and causing a high rise of blood pressure. Dilatations of the internal sphincter were performed and the patient was placed on alpha-blocking drugs but with no benefit. Radiological studies showed a posterior tilting of the bladder, and the patient had a prostato-cysto-pexy and a bladder neck resection. Accidentally, during the operation, the bladder dome was perforated, and required to be sutured, and it was necessary to use the pyramidalis muscle brought backwards so as to fix the prostate to avoid the risk of pubic osteitis (the urine was infected) (Fig. 3a, b, c, d). The result was quite remarkable. The patient when seen in 1986, empties his bladder completely in twenty seconds and this is six years after the operation. A few intermittent urinary infections have occurred. The perineum remains denervated and the bladder wall still tends to tilt backwards. No residual urine was found after micturition, and the urine is sterile. 


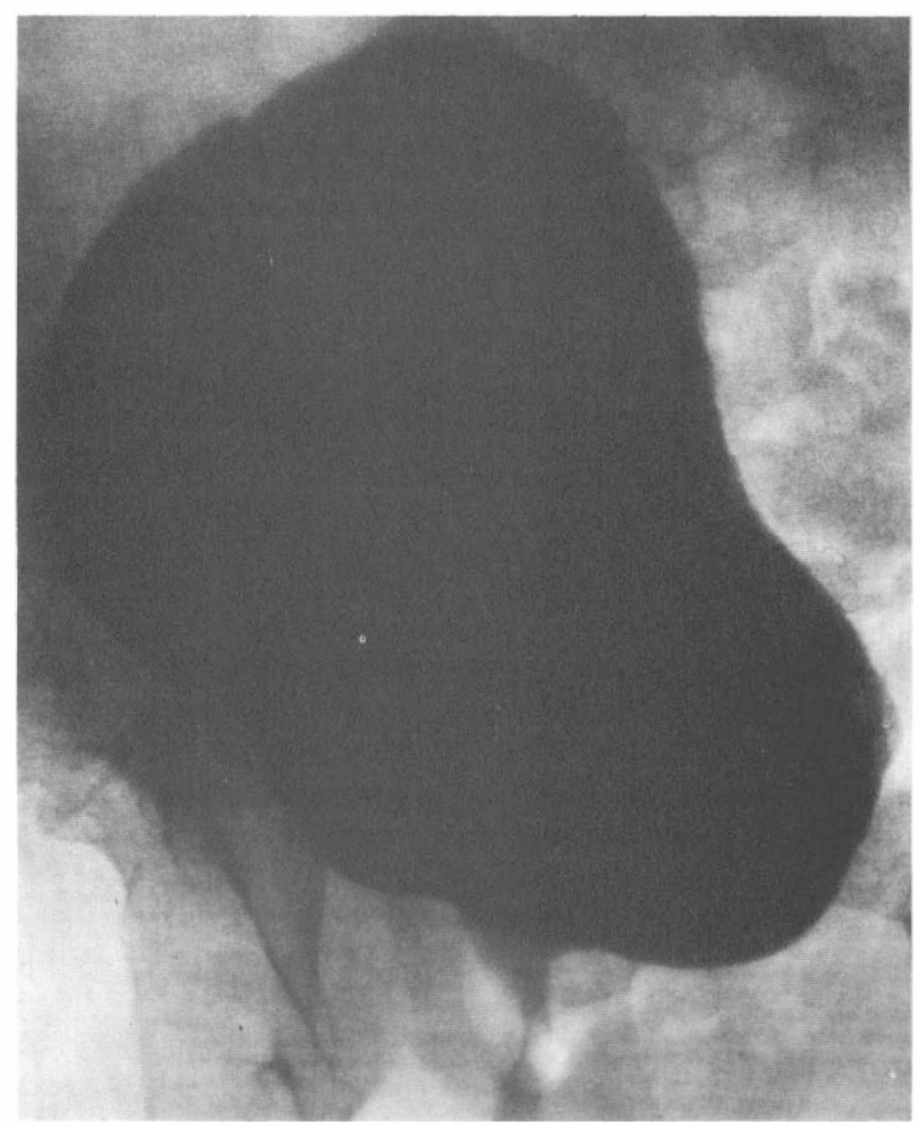

Figure 4 Voding cysto-urethrogram, in 1979, 8 years after the first operation (published in Paraplegia, Dollfus et al., 1972). No residual urine.

Case 3 Ne... R.: a fracture of L1 vertebra occurred at the age of 51, in 1973, causing an incomplete lower motor neuron paraplegia below T9, complete below $\mathrm{T} 12$ on the left, and L 1 on the right. Intermittent catheterisation was instituted, followed by a resection of the bladder neck. Thereafter, he could micturate easily by abdominal strain. Ten years later, voiding difficulties occurred, and in January 1985 a prostato-cysto-pexy, using the pyramidalis muscle, was performed, associated with a resection of a small prostatic adenoma. Since then there have been no urinary problems, and micturition occurs without difficulty by abdominal straining, and there is no residual urine. When seen in 1986 the result of treatment was good, and the urine was sterile.

Case $4 \mathrm{Fe} \ldots \mathrm{D}$.: this patient in 1966 sustained a fracture-luxation of the L1 vertebra on L2, at the age of 17 , causing a conus medullaris and cauda equina lesion, clinically incomplete, motor and sensory, below L5. At first, micturition was possible by abdominal straining after bladder training. Sixteen years later, the bladder and the perineum was still of the lower motor neuron type, micturition became inefficient, even during intense abdominal straining, and urinary infections became more and more frequent. The diagnosis of a dynamic 
tilting of the prostate was followed by a prostato cysto pexy operation in 1984, with fixation of the prostate to the pyramidalis muscle. Two years after the operation, follow up studies were performed, and these showed that micturition was easily achieved without residual urine, with marked improvement of his occasional stress incontinence, and the urine was sterile.

Four similar operations, performed on paraplegics of traumatic origin with an inactive bladder and perineum were followed by encouraging results. These cases, in our opinion, can demonstrate the reality of this late, but rare complication, occurring in patients with lower motor neuron lesions and, in one patient with a mixed upper and lower motor neuron lesion, incomplete below L3, operated in 1980, eight years after the accident.

We have previously published (Jurascheck et al., 1980) the case of an upper motor neuron lesion, which showed a similar phenomenon by rupture of the pubourethral ligaments. This person was not operated on as satisfactory micturition had been obtained by bladder re-training, using other methods to assist micturition.

\section{Discussion}

The literature on these particular static and dynamic changes, causing an increasing dysuria in patients with a neuropathic bladder is found mainly in French literature (see below).

We wish to quote J. Hutch (1972) referring to flaccid neuropathic bladders: '... the portion of the urethra passing through the urogenital diaphragm does not share in the generalised urethral dilation and appears to be obstructive.' This obstruction is enhanced by the mechanism we have described. The post operative results seem to confirm this hypothesis.

The second operative method, using the pyramidalis muscle appears to be preferable, as there is no risk of infecting the pubis, using a non resorbing suture, even with sterile urine. The absence of an intervention on the recto vesical recess, is associated with the anchoring of the sigmoid colon upwards so as to give posterior support to the bladder (Archimbaud, 1974, Archimbaud et al., 1976). This technique could have been useful but none of our patients had any complications from not using it. Furthermore, there is a risk of interference of the intestine, which is already very perturbed in patients with lower motor neuron lesions. Self catheterisation has been used as a temporary measure, advised as an alternate conservative method but refused by these patients most of whom had been successfully bladder trained, and wanted to keep their usual voiding habits. Recurrence of dysuria in our patients, after the interventions, has always been caused by new additional pathologies, either a urethral stenosis or a prostatic adenoma.

\section{Conclusion}

Patients with functional dysuria, due to the backwards tilting on the prostate bladder block, in lower, or mixed lower and upper motor neuron lesions, during micturition by intense straining can be offered a surgical treatment by a prostato- 
cysto-pexy. This operation was performed in eight patients with very encouraging results.

\section{References}

ARChimbaud JP 1974 Les dysfonctionnements vésico-sphinctériens neurologiques. Rapports du 68e Congrès de l'Association Française d'Urologie. Paris, Masson, pp. 129-135.

ARChimbaud JP, Spay G, BuZelin J, et al. 1976 La bascule postérieure de la vessie et de l'urètre de certaines neurovessies périphériques et son traitement par la colocystopexie. fournal d'Urologie et de Néphrologie 82:231-239.

BuZelin JM, JuRASCHECK F 1981 Les complications urologiques et leur traitement. La Paraplégie (Maury M.). Paris, Flammarion Medecine-Sciences, pp. 334-344.

Dollfus P, JuRASChECK F, JACOB-Chia D et al. 1972 Prostato-cystopexy for a paralegic case with an inactive bladder and perineum. Paraplegia 10:64-67.

Hutch JA 1972 Anatomy and physiology of the bladder, trigone, and urethra. London, Butterworths, pp. 139-140.

Jurascheck F, Dollfus P, Mennecier A, et al. 1974 Problèmes posés par les paraplégies flasques des muscles du périnée. Procês-verbaux, mémoires et discussions. Assocation Française d'Urologie. Paris, Masson, pp. 298-302.

JuRASCHECK F, Dollfus P, JACOB-Chia D 1980 Elongation of the active anterior wall of the urogenital pelvic diaphragm, a late unusual complication of paraplegia. Paraplegia 18:241-244.

JuRASCHECK F, ChAPUIS A, FERNANDEZ R, et al. 1981 Les dysuries par modification de la statique périnéale dans les lésions neurologiques. Société Internationale Francophone d'UroDynamique. Rapport du 3e Congrès, Rome, pp. 181-191. 